\title{
MYOPATHY IN CUSHING'S SYNDROME
}

\author{
BY \\ RAGNAR MÜLLER and ERIC KUGELBERG

\begin{abstract}
From the Department of Neurology, Serafimerlasarettet, and the Department of Endocrinology, Karolinska Sjukhuset, Karolinska Institutet, Stockholm, Sweden
\end{abstract}

Muscular weakness is a well-known feature of Cushing's syndrome and was mentioned by Cushing (1932a and b) in his original description of the disease. It has been suggested that the muscular weakness and the atrophy sometimes occurring may be due to increased breakdown of proteins (Soffer, 1951) or to decreased protein synthesis (Lukens, 1954).

So far as we are aware, no systematic investigation of the occurrence and extent of the paresis in Cushing's syndrome has been reported in the literature. Neither do morphological alterations in the muscle appear to have been studied. Marburg (1933) reported a case of Cushing's syndrome with weakness of the pelvic girdle musculature in which necropsy revealed histological changes of the muscular dystrophy type. He pointed out, however, that in this case there was a combination of Cushing's syndrome and progressive muscular dystrophy. Accordingly, the pathological process underlying the muscular weakness in Cushing's syndrome is unknown.

We have studied the motor function in six patients with typical Cushing's syndrome clinically and electromyographically. Muscle biopsy was done in four of them.

Five of the patients had weakness involving primarily the proximal muscles of the lower limbs, and in them the electromyographic examination revealed signs of myopathy. The histological studies disclosed degeneration of muscle fibres.

\section{Case Reports}

Case 1.-A woman, born in 1916, developed the classical features of Cushing's syndrome in 1950. Since 1957 she had had weakness of the legs, had difficulty in climbing stairs, and was unable to mount a ladder. She had also noticed some difficulty in raising her arms over the head. She had not received any treatment.

Neurological Examination (October, 1958).-There was moderate weakness of dorsiflexion of the feet and toes and considerable weakness of extension at the knees and of flexion, extension, and adduction at the hips. Trendelenburg's test was positive bilaterally. She was unable to step up on to a chair or to rise from the squatting position without the aid of her hands. She was unable to walk on her heels. There was slight weakness of forward flexion and abduction at the shoulders. There was no definite atrophy. The muscle reflexes were normal. The plantar responses were flexor. Sensation was unimpaired.

Electromyographic Examination.-Examination of the quadriceps and anterior tibial and deltoid muscles revealed signs of myopathy, most pronounced in the quadriceps.

Histological Report.-Transverse sections of the right quadriceps muscle showed slight infiltration of fat cells between the muscle bundles. The connective tissue was moderately increased and in one place dense and hyaline. The endomysial connective tissue was also slightly increased in places. Most of the muscle fibres were somewhat thin, but as a rule the polygonal contour was retained. At the edge of one muscle bundle was seen a group of rounded fibres which were hyalinized and somewhat larger than the other fibres. The striation was preserved in most areas. Most of the sarcolemmal nuclei had retained their shape and hypolemmal position; some nuclei were small, dark, and elongated and relatively increased in number. Occasional lymphocytes were present in the interstitial tissue. The blood vessels and nerves were normal.

Case 2.-A man, born in 1939, in whom a typical Cushing syndrome developed in 1953 . Since 1956 he had noticed slight weakness of the legs. Bilateral adrenalectomy was performed in two stages in August and September of 1958. The right adrenal showed a moderate cortical hypertrophy, the left adrenal was normal. After the operation he had received $50 \mathrm{mg}$. of cortisone daily.

Neurological Examination (October, 1958).- There was slight weakness of extension at the left knee and of all movements at the hips. Trendelenburg's test was negative. There was no weakness of the arms. There was no evidence of muscular atrophy. The muscle reflexes were normal. The plantar responses were flexor. Sensation was unimpaired.

Electromyographic Examination.-Examination of the quadriceps and anterior tibial muscles revealed signs of myopathy, most pronounced in the quadriceps.

Case 3.-A woman, born in 1925, developed Cushing's syndrome in 1950, rapidly increasing in severity. The 
disease was accompanied by progressive weakness of the legs. She had difficulty in walking and frequently fell down. At the end of 1953 a right subtotal adrenalectomy was performed and a cortical adenoma the size of a large pea was resected. After the operation she had received $50 \mathrm{mg}$. of cortisone daily. During the following year the physical abnormalities practically disappeared, but an extremely severe pigmentation appeared over the entire body and on the mucous membranes. Power in the legs was slightly increased.

Neurological Examination (October, 1958). - There was moderate weakness of extension at the knees and of flexion and adduction at the hips. She was unable to step up onto a chair with the left foot and only with difficulty with the right foot without the aid of her hands. She could only just rise from the squatting position. Trendelenburg's test was negative. There was no weakness of the arms. There was no evidence of muscular atrophy. The muscle reflexes were normal. The plantar responses were flexor. Sensation was unimpaired.

Electromyographic Examination.-Examination of the quadriceps, anterior tibial, deltoid, and first dorsal interosseous muscles revealed signs of myopathy, most pronounced in the quadriceps.

Histological Report.-Most of the muscle bundles of the right quadriceps had normal configuration. They were separated by slightly increased amounts of connective tissue and fat. A number of degenerated fibres were seen lying in groups but also singly among normal muscle fibres. Some of them were comparatively large. A few fibres were completely hyalinized. The sarcolemmal nuclei had for the most part retained their normal shape and hypolemmal position; some were small, dark, and arranged in longitudinal rows and relatively increased in number. There were no inflammatory cells. The blood vessels and nerves were normal.

Case 4.-A woman, born in 1918, since 1954 had presented the signs and symptoms of Cushing's syndrome, including weakness of the legs, most pronounced when climbing stairs. Earlier her muscles had been strong and she had taken part in athletic sports. In May, 1958, a basophilic hypophyseal adenoma was extirpated after which the Cushing symptoms largely disappeared. After the operation she received $25 \mathrm{mg}$. of cortisone daily. The weakness of the legs, however, persisted.

Neurological Examination (October, 1958).- There was moderate weakness of extension at the knees and slight weakness of all movements at the hips, particularly of flexion, abduction, and adduction. She had great difficulty in stepping up onto a chair and rising from the squatting position without the aid of her hands. Trendelenburg's test was negative. There was no weakness of the arms. There was no evidence of muscular atrophy. The muscle reflexes were normal. The plantar responses were flexor. Sensation was unimpaired.

Electromyographic Examination.-Examination of the quadriceps muscles revealed signs of myopathy.

Histological Report.-In some areas of the right quadriceps the muscle fibres were replaced by dense hyaline connective tissue and to a lesser degree by fat.
In places the endomysial connective tissue was also slightly thickened. The diameter of the fibres varied: many were normal, others thin or slightly hypertrophic. In cross sections the fibres usually appeared rounded. In most of the fibres striation was intact. Most of the sarcolemmal nuclei had retained their normal shape and position: some, especially in the thin fibres, were small, dark, and occasionally arranged in longitudinal rows; some had migrated centrally. Isolated fibres were hyalinized and contained dark, shrunken nuclei which sometimes lay in clumps. In places single or rows of pyknotic sarcolemmal nuclei lay free in the connective tissue or close to thin degenerated fibres. In one area there was a suggestion of regeneration. There were no inflammatory cells. The blood vessels and nerves were normal.

Case 5.-A woman, born in 1906, developed the classical features of Cushing's syndrome in the late thirties and they steadily progressed. In 1945, she received a course of $x$-ray irradiation to the pituitary with temporary improvement. In 1950, she was given a second course of $x$-ray irradiation and was placed on testosterone propionate. During the next two years the symptoms regressed but since 1952 her condition had remained comparatively stationary with mild, but distinct signs of the Cushing syndrome. Since 1948 she had noticed weakness of the legs and difficulty in climbing stairs. There was also slight loss of power in the arms, manifested chiefly by difficulty in lifting heavy objects.

Neurological Examination (October, 1958). - There was slight weakness of dorsiflexion of the feet and toes and moderate weakness of extension at the knees and of flexion at the hips. She was unable to mount a chair or rise from the squatting posture without the aid of her hands. Trendelenburg's test was negative. There was slight weakness of forward flexion and abduction at the shoulders. There was no muscular atrophy. The right quadriceps reflex was weaker than the left. All other muscle reflexes were normal and bilaterally equal. The plantar responses were flexor. Sensation was unimpaired.

Electromyographic Examination.-Examination of the quadriceps, anterior tibial, and deltoid muscles revealed signs of myopathy, most pronounced in the quadriceps.

Histological Report.-In places in the left quadriceps the muscle fibres were replaced by fat and, to a lesser degree, by connective tissue. Most of the fibres were slightly thinner than normal but had retained their polygonal shape and striation. A few fibres, however, were hyalinized and striation obscured. Many of the sarcolemmal nuclei were small, dark, and elongated. In the thin fibres the nuclei were relatively increased and sometimes arranged in longitudinal rows; a few were centrally placed. The interstitial tissue contained a few lymphocytes. The blood vessels and nerves were normal.

Case 6.-A woman, born in 1912, since 1953 had presented the clinical features of Cushing's syndrome. In February, 1955, the right suprarenal gland was removed and was found to contain a zona reticularis tumour the size of a walnut. After the operation all signs and symptoms of Cushing's syndrome disappeared. She had never noticed any weakness of the limbs. 
Neurological Examination (October, 1958).-There was no evidence of muscular weakness or wasting. The muscle reflexes were normal. The plantar responses were flexor. Sensation was normal.

Electromyograms from the quadriceps muscles were normal.

\section{Results}

Muscular Signs and Symptoms.-Five patients had weakness of the muscles of the pelvic girdle and thighs. In two of them (Cases 3 and 4 ) this was one of the first symptoms of the disease and in both had progressed rapidly. In three patients (Cases 1, 2, and 5) the muscular weakness began three, seven, and 10 years, respectively, after the onset of the disease, when the other symptoms were severe, and had developed slowly, becoming marked in two of them. Case 6 had no muscular weakness.

Case 1 had a slight waddling gait and a positive Trendelenburg's test bilaterally. The remaining patients had a normal gait. Four patients (Cases 1, 3,4 , and 5) were unable to step up onto a chair or rise from the squatting position without the aid of their hands.

Two patients (Cases 1 and 5) also had weakness of the muscles of the shoulder girdle and of dorsiflexion of the feet. In both, this had developed later and was less pronounced than the weakness in the lower limbs. The abdominal, neck, or facial muscles were not involved.

None of the patients had muscular pain or tenderness. There was no definite atrophy and no evidence of pseudohypertrophy. Muscular fasciculations were not observed.

One patient (Case 5) had unilateral impairment of the quadriceps reflex. The muscle reflexes were otherwise normal. The plantar responses were always flexor. There were no sensory changes.

In three patients (Cases 3, 4, and 5) treatment had resulted in partial or complete regression of the Cushing syndrome. The muscular weakness, however, persisted, although in Case 3 there was a slight improvement of muscle power.

Electromyography.-None of the electromyograms showed spontaneous fibrillation potentials or positive sharp waves. The insertion activity was normal. There were no signs of increased muscular irritability or denervation.

During maximal voluntary contraction a large number of action potentials were recorded, producing the normal interference pattern. Accordingly, there was no gross reduction in the number of motor units.

The appearance of the individual potentials, however, was altered in five patients. This abnormality consisted of a general reduction in the size and duration of the individual potentials and an in-

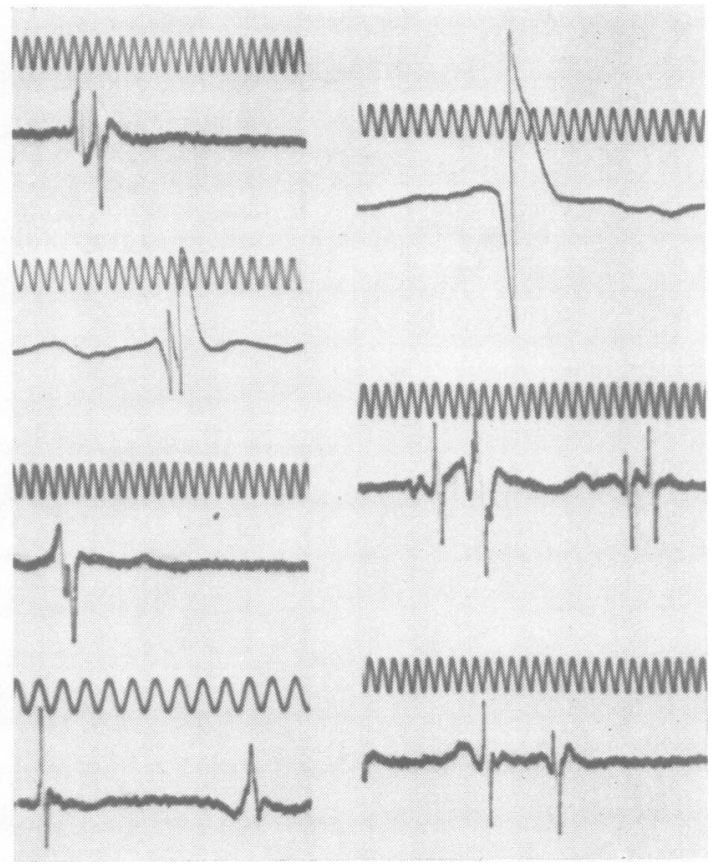

Fig. 1.-Case 1. Eleven consecutive action potentials recorded from the right quadriceps muscle. The majority have a duration of about $3 \mathrm{~m}$. sec. Two polyphasic potentials are seen at the bottom right hand side. Time: $1 \mathrm{~m}$. sec.

creased proportion of polyphasic potentials. The changes were most marked in Case 1 , in which the paresis was also most severe. In Case 6 the electromyogram was normal. This patient had no clinical signs of paresis. Fig. 1 shows the appearance of the individual action potentials in Case 1. Fig. 2 shows

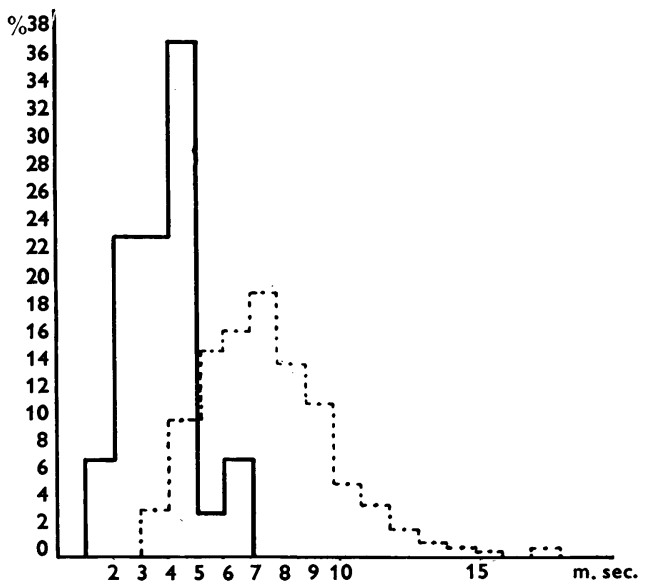

Fig. 2.-Distribution of the duration of 30 action potentials recorded from the quadriceps muscle in Case 1 (solid line) and 300 action potentials recorded from the biceps muscle in healthy individuals (broken line). 


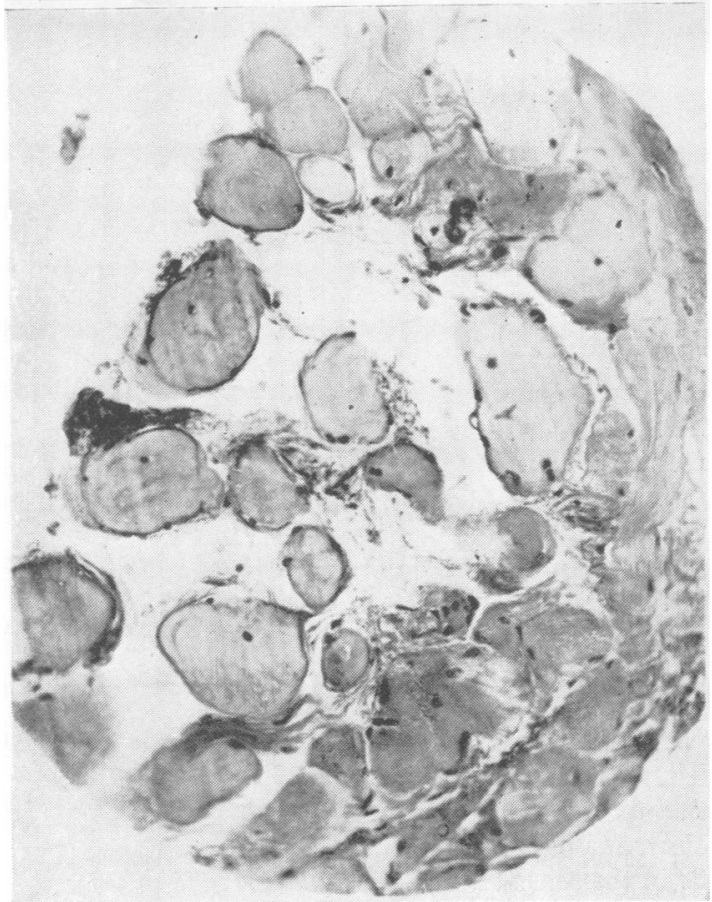

FIG. 3

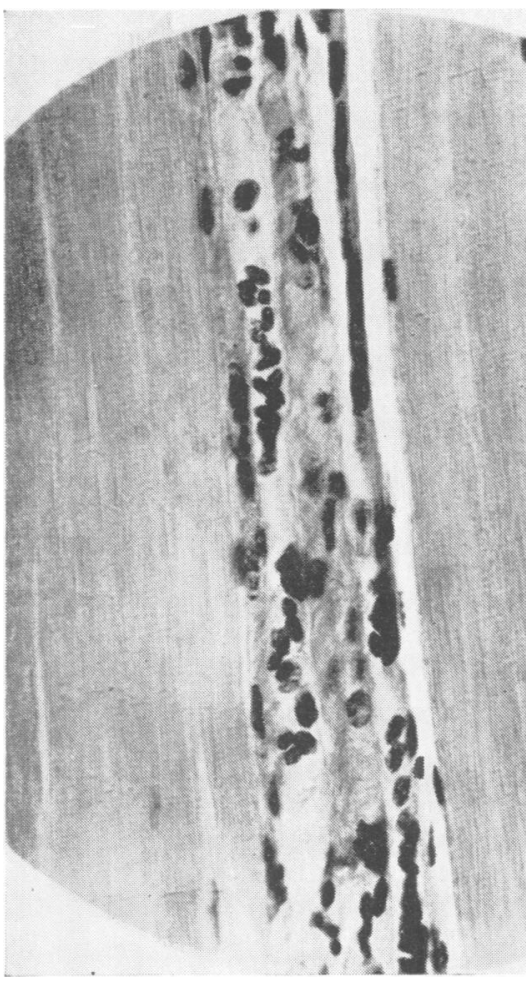

FIG. 5

Fig. 3.-Case 4. Section of biopsy from quadriceps muscle. Scattered large as well as small degenerated fibres. A few sarcolemmal nuclei are centrally placed. Heidenhain-Van Gieson. $\times 200$

Fig. 5.-Case 4. Section of biopsy from quadriceps muscle. A group of extremely thin, partially fragmented muscle fibres with increased number of sarcolemmal nuclei is seen between two groups of unaffected fibres. Hansen-Van Gieson. $\times 375$.

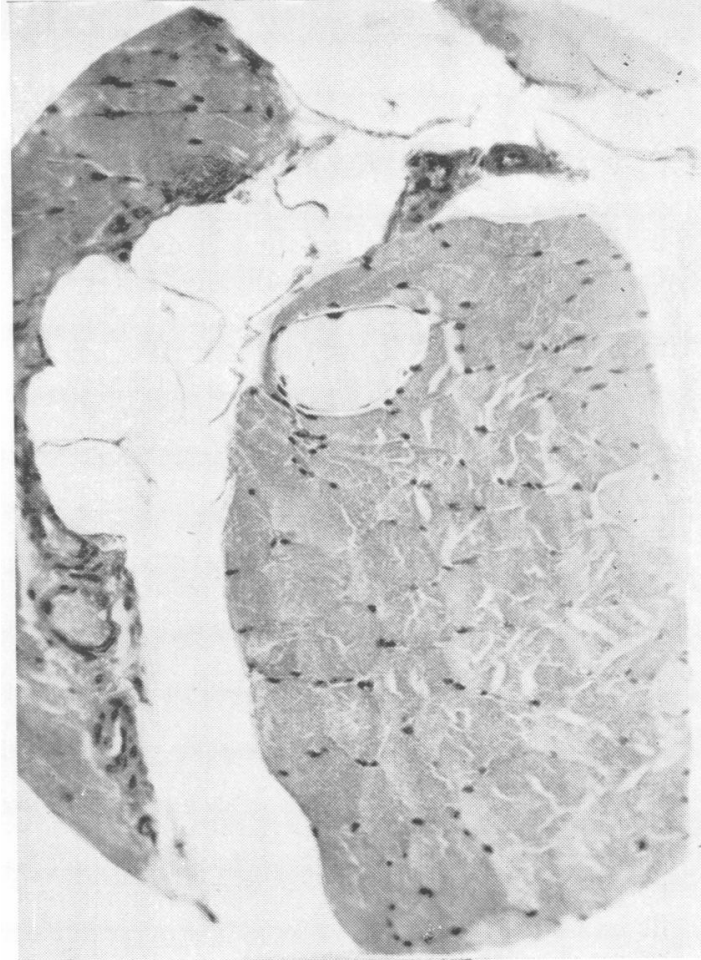

FIG. 4

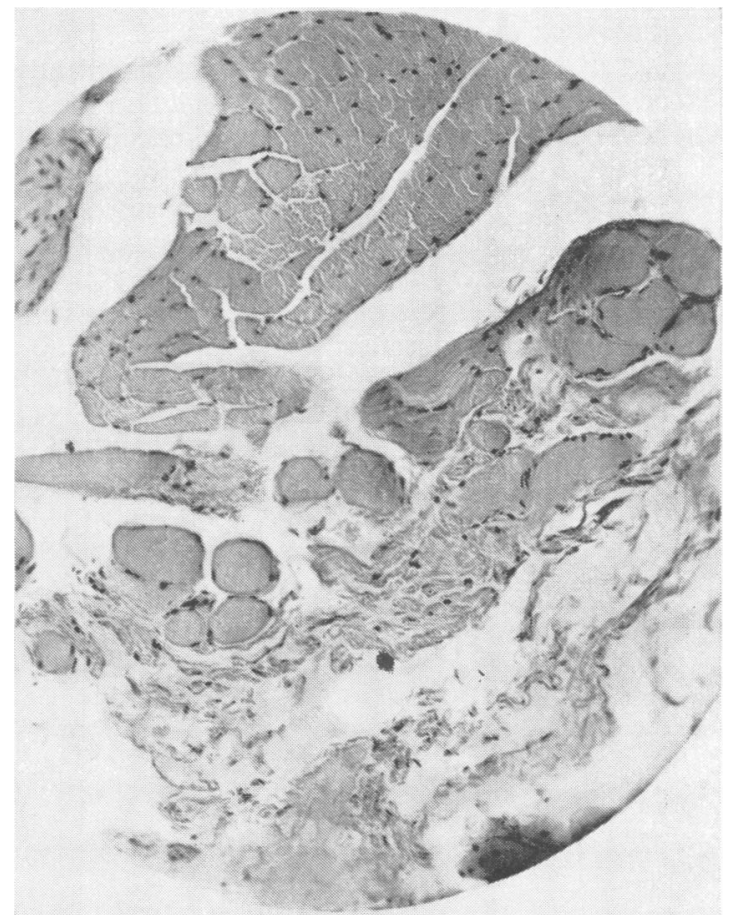

FIG. 6

Fig. 4-Case 4. Section of biopsy from quadriceps muscle. A solitary swollen, hyalinized fibre at the edge of a bundle of normal muscle fibres is separated from adjacent normal muscle bundles by an increased amount of fatty tissue. Heidenhain-Van Gieson. $\times 200$.

Fig. 6.-Case 1. Section of a biopsy from the quadriceps muscle. Hyalinized fibres of varied size adjacent to a field of normal fibres. The connective tissue is increased. No evidence of interstitial inflammatory changes. Hansen-Van Gieson. $\times 135$. 
the duration of action potentials from the quadriceps muscle of Case 1 compared with that in normal biceps muscles (Petersén and Kugelberg, 1949). Limited studies in healthy individuals show that the duration of the action potentials in the quadriceps muscle lies within the limits of normal values for the biceps muscle.

The anterior tibial muscle in Case 1 also showed marked electromyographic changes. Slight electromyographic changes were seen in distal limb muscles which exhibited no clinical paresis, as, for example, the first dorsal interosseus of the hand in Case 3. The most pronounced changes, however, were always found in the proximal muscles of the legs, which also showed the most severe muscular weakness.

Histological Examination.-The histological picture was the same in all the four cases examined and showed pathological changes (Figs. 3 to 6).

The muscle fibres were to a small extent replaced by connective tissue and fat. The diameter of the fibres varied. The majority of the fibres were somewhat thin but had otherwise normal structure. Hypertrophic fibres were also encountered. All specimens contained degenerated fibres lying in groups or singly among normal muscle fibres. The degenerated fibres usually had a rounded contour and were hyalinized and the striation obscured. Most of them were of normal thickness or enlarged. In Case 4 foci of extremely thin, fragmented fibres were seen. Most of the sarcolemmal nuclei had retained their shape and hypolemmal position. Some were dark, small and elongated, increased in number, and sometimes arranged in longitudinal rows. Occasional nuclei had migrated centrally. In Case 4 clumps of pyknotic sarcolemmal nuclei were lying free in the connective tissue. There was no convincing evidence of regeneration. Inflammatory changes did not occur. The blood vessels and nerves were normal.

In no case were the abnormalities severe. The changes were most marked in Case 4 , and least in Cases 1 and 5, in which the degree of severity was appproximately the same. Case 1 , however, had the most pronounced paresis and the most distinct electromyographic changes, while in Case 4 the paresis was less pronounced and the electromyographic changes less severe.

\section{Discussion}

Five of the six patients had paresis involving primarily the muscles of the pelvic girdle and thighs. Pareses due to neurogenic lesions have been reported in endocrine disorders such as acromegaly by, among others, Woltman and Kernohan (1955). A neurogenic origin of the muscular weakness in Cushing's syndrome is improbable in view of the proximal distribution of the pareses, the absence of fasciculations, the preservation of muscle reflexes, and the intact sensibility. Neither did the electromyographic and histological studies reveal any evidence of neurogenic lesions. The cause of the muscular weakness in Cushing's syndrome probably must primarily be sought in the muscle itself. This may then be a metabolic disorder which decreases the power of contraction of the individual muscle fibres or a numerical reduction of functioning fibres in the motor units.

The electromyograms showed a decrease in the duration of individual action potentials and an increased proportion of polyphasic potentials. This pattern is characteristic of disorders in which there is a reduction in the number of functioning fibres in the motor units (Kugelberg, 1947, 1949). Such electromyographic changes are, however, not specific in the sense that they may be expected to occur in any disease in which there is disintegration of the motor units, irrespective of whether this is due to reversible functional disturbances or to morphological muscle changes (Kugelberg, 1949). On the other hand, histological examination in our cases revealed the presence of varying degrees of degeneration of the muscle fibres. Consequently, in Cushing's syndrome it is a question of a true myopathy.

The histological changes were less pronounced, however, than might be expected from the clinical and electromyographic observations. In this respect the myopathy in Cushing's syndrome resembles thyrotoxic myopathy in which severe muscular weakness is not necessarily associated with pronounced morphological changes in the muscles (Adams, Denny-Brown, and Pearson, 1953; Hed, Kirstein, and Lundmark, 1958). One explanation of the discrepancy may be that the muscular weakness in Cushing's syndrome is due in part to electrolyte disturbances. This seems less probable, however, since in our patients the pareses persisted unchanged over long periods. Neither was there any definite improvement in muscle power when treatment had resulted in partial or complete regression of the other symptoms. The discrepancy may, of course, be due to the biopsy specimens not being representative of the affected muscle. A further possibility is that the routine histological techniques used were inadequate to visualize insignificant morphological changes in fibres put out of function.

The myopathy in Cushing's syndrome may be related to the increased production of corticoids. Muscle changes have been induced in experimental animals with cortisone and A.C.T.H. (Germuth, Nedzel, Ottinger, and Oyama, 1951). In rabbits 
daily administration of cortisone at a dosage of $10 \mathrm{mg}$. per $\mathrm{kg}$. body weight rapidly produces varying amounts of segmental degeneration of muscle fibres which is reversible (Ellis, 1956; Glaser and Stark, 1958). Cortisone-induced myopathy cannot be prevented by the administration of potassium (Ellis, 1956).

In the cases of Cushing's syndrome studied here the clinical manifestations of myopathy showed little tendency to be reversible. Neither were there any convincing signs of regeneration of muscle fibres in the biopsy specimens. The muscle changes were considerably less pronounced than those reported in experimental cortisone myopathy which, histologically, gives the impression of being an acute myopathy.

Before treatment the patients were, roughly estimated, producing from 100 to $150 \mathrm{mg}$. of corticoids per day, i.e., about $2 \mathrm{mg}$. per $\mathrm{kg}$. body weight. This amount is small compared with that administered experimentally, but while the animal experiments lasted only a few weeks, the patients were exposed to the effect of increased corticoid production for years. The difference between the myopathy in Cushing's syndrome and cortisone-induced myopathy should be considered against this background.

It is known that prolonged cortisone medication in large doses may lead to severe muscular weakness (Lukens, 1954, among others). It is possible that the muscular weakness in these cases is due to myopathy of the same type as we observed in Cushing's syndrome.

\section{Summary}

In six patients with Cushing's syndrome the muscle functions have been studied clinically and electromyographically. Muscle biopsy was done in four of them.

Five patients had weakness of the proximal muscles of the lower limbs; two of them had, in addition, weakness of the shoulder girdle muscles and of the dorsiflexors of the feet. The paresis was most pronounced in the proximal muscles of the lower limbs. Only in one case was the paresis severe; in the other four it was moderate or slight. One patient had no muscular weakness.

In three of the patients the muscular weakness appeared several years after the onset of the disease, when the other symptoms had become severe. After successful treatment of the Cushing syndrome the muscular weakness persisted unchanged for years in two cases; in one case there was a slight increase of muscular power.

The electromyographic examination in the five patients with paresis revealed signs of myopathy, most pronounced in the muscles that were clinically weakest. Changes of the myopathic type were also observed in distal limb muscles which clinically were completely normal.

The histological changes consisted of slight or moderate degeneration of muscle fibres. The intensity of the changes was less than might be expected from the clinical and electromyographic observations.

It is concluded that the muscular weakness in Cushing's syndrome is due to a true myopathy, probably produced by the increased production of corticoids.*

We are indebted to Professor Nils Antoni and Assistant Professor Karl Erik Åström for the histological examinations.

\section{REFERENCES}

Adams, R. D., Denny-Brown, D., and Pearson, C. M. (1953). Diseases of Muscle. Hoeber, New York.

Cushing, H. (1932a). Bull. Johns Hopkins Hosp., 50, 137.

(1932b). J. Amer. med. Ass., 99, 281.

Ellis, J. T. (1956). Amer. J. Path., 32, 993.

Germuth, F. G., Nedzel, G. A., Ötinger, B., and Oyama, J. (1951). Proc. Soc. exp. Biol. (N.Y.), 76, 117 .

Glaser, G. H., and Stark, L. (1958). Neurology, 8, 640.

Hed, R., Kirstein, L., and Lundmark, C. (1958). J. Neurol. Neurosurg. Psychiat., 21, 270.

Kugelberg, E. (1947). Ibid., 10, 122.

Kugelberg, E. (1947), Ibid.,

Lukens, F. D. W. (1954). Medical Uses of Cortisone. Blakiston, New York-Toronto.

Marburg, O. (1933). Arb. neurol. Inst. Univ. Wien., 35, 143.

Petersén, I., and Kugelberg, E. (1949). J. Neurol. Neurosurg. Psychiat.. 12, 124.

Soffer, L. J. (1951). Diseases of the Endocrine Glands. Lea \& Febiger, Philadelphia.

Woltman, H. W., and Kernohan, J. W. (1955). In Baker, A. B., Clinical Neurology, Vol. 3, p. 1563. Hoeber, New York.

* After this paper was sent in for publication another patient with an untreated Cushing's syndrome, who exhibited much more severe myopathic changes, was seen in the Clinic. 